Sibylle Peters/Norbert Bensel (Hrsg.)

Frauen und Männer im Management 
Sibylle Peters/Norbert Bensel (Hrsg.)

\section{Frauen und Männer im Management}

Diversity in Diskurs und Praxis

2., überarbeitete und erweiterte Auflage

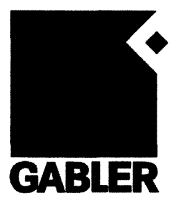


Die Deutsche Bibliothek - CIP-Einheitsaufnahme

Ein Titeldatensatz für diese Publikation ist bei

Der Deutschen Bibliothek erhältlich

Prof. Dr. Sibylle Peters lehrt berufliche und betriebliche Weiterbildung an der Fakultät

für Geistes-, Sozial- und Erziehungswissenschaften der Otto-von-Guericke-Universität Magdeburg.

Dr. Norbert Bensel ist Mitglied des Vorstands der DaimlerChrysler Services AG Berlin und ist in dieser Position verantwortlich für das Ressort Personal.

1. Auflage September 2000

2. Auflage April 2002

Alle Rechte vorbehalten

(c) Betriebswirtschaftlicher Verlag Dr. Th. Gabler GmbH, Wiesbaden 2002

Softcover reprint of the hardcover 2nd edition 2002

Lektorat: Barbara Roscher / Renate Schilling

Der Gabler Verlag ist ein Unternehmen der Fachverlagsgruppe BertelsmannSpringer. www.gabler.de

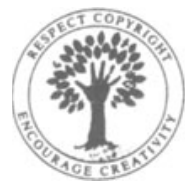

Das Werk einschließlich aller seiner Teile ist urheberrechtlich geschützt. Jede Verwertung außerhalb der engen Grenzen des Urheberrechtsgesetzes ist ohne Zustimmung des Verlags unzulässig und strafbar. Das gilt insbesondere für Vervielfältigungen, Übersetzungen, Mikroverfilmungen und die Einspeicherung und Verarbeitung in elektronischen Systemen.

Die Wiedergabe von Gebrauchsnamen, Handelsnamen, Warenbezeichnungen usw. in diesem Werk berechtigt auch ohne besondere Kennzeichnung nicht zu der Annahme, dass solche Namen im Sinne der Warenzeichen- und Markenschutz-Gesetzgebung als frei zu betrachten wären und daher von jedermann benutzt werden dürften.

Umschlaggestaltung: Ulrike Weigel, www.CorporateDesignGroup.de

Gedruckt auf säurefreiem und chlorfrei gebleichtem Papier

ISBN-13: $978-3-409-21638-8$

e-ISBN-13: 978-3-322-82868-2

DOI: $10.1007 / 978-3-322-82868-2$ 


\section{Editorial}

\section{Was können Sie von diesem Buch erwarten?}

Vielfältige Veränderungen und Formen von Transformationsprozessen spiegeln sich in diversen Diskursen der Risikogesellschaft wider. Ständig entstehen in immer komplexer werdenden strukturellen Kopplungen auf immer höherem Niveau neue Steuerungsmodelle zur Neuaushandlung und Neudispositionierung. Ganz allgemein geht es um theoretische und praktische Versuche, die instabile Hoch-Moderne reflexiv zu verstehen und neue Optionen für „vernünftige“ Gestaltungsaufgaben zur Bewältigung von Zukunft zugänglich zu machen. In diesen vielfältigen gesellschaftlichen Diskursarten ist die Diskussion über Geschlechterdifferenz und -arrangements als ein Teilbereich eingebunden, wie z.B. Veröffentlichungen zum Thema Frauen und Männer in Führungspositionen aufweisen, die auf dem Markt verstärkt vertreten sind.

Das Thema Frauen in Führungspositionen ist in den letzten Jahren von zahlreichen „Wegbereitern“ und „Treibern“ gesellschaftspolitischer Veränderungsprozesse in Gesellschaft und Wirtschaft in diversen Modernisierungsexpertisen vorangetrieben worden. Diese Diskussionsprozesse und Rechtsexpertisen in Europa und den USA haben wesentlich dazu beigetragen, dass Gender-Mainstreaming, Managing-Diversity und ChangeManagement drei der wichtigsten aktuellen Managementstrategien sind und das Thema Frauen in Führungspositionen als wesentlicher Teilbereich von Organisationsentwicklung nicht mehr wegzudenken ist. Im Rahmen der Umstrukturierungen in Wirtschaft und Verwaltung gilt für die oben genannten Strategien gleichermaßen, dass Frauenförderung in Führungspositionen keine „Frauensache“ ist, sondern im internationalen Kontext in diverse globale Modernisierungsstrategien eingebunden ist.

Der Band widmet sich dem besonderen Fokus von Diversity, der innerhalb von nationalen Grenzen und auf europäischem Raum jedoch nicht ohne die Expertise GenderMainstreaming und den besonderen Organisationsentwicklungsformen in Recht und Verwaltung gesehen und verstanden werden kann. Diversity steht derzeit vorwiegend in globalisierten Unternehmen zur Disposition. Ihr Transfer ist für heterogene Beschäftigten- und Mitarbeitergruppierungen besonders interessant, da dieser Ansatz die Wahrnehmung der Sekundärkriterien wie Lifestyle, Bildung, Sprache und normative Wertevorstellungen in ihrem Wandel in globalen wie lokalen Färbungen aufnehmen kann und dadurch für viele diverse Transformationen Anregungen für den Diskurs in Theorie und Praxis bietet. Der Diskurs zu Fragen von Gleichstellung und Chancengleichheit trifft als Zeitgeist auf einen Strukturwandel, der sich zunehmend auf Fragen nicht-ökonomischer Austauschprozesse konzentriert und soziale-interaktive Formen des Austausches inner- 
Editorial

halb des ökonomischen Geldtransfers wichtiger werden und zunehmend Beachtung finden lässt. Frauen in Führungspositionen, die neben Männern und mit Männern die Zukunft gemeinsam gestalten, werden für Wettbewerbsaspekte immer wichtiger.

Der Diskurs enthält etliche plurale Formen und spiegelt innerhalb verschiedener Perspektiven jeweils unterschiedliche Entwicklungen wider. In ihrer Gesamtheit lassen sich die Beiträge von dem Gedanken leiten, einen Diskurs im Sinne des Suchens nach einem „vernünftigen“ Umgang mit ständig neuen Formen des Verhältnisses von Arbeit, Leben und Lernen zu ermöglichen. Insbesondere wird die Möglichkeit thematisiert, dass das „standardisierte“ Normalarbeitsverhältnis des Mannes, in dem unhinterfragt die Karriereplanung nur in einer spezifischen Monokausalität vernünftig ist und Vernünftigkeit beanspruchen konnte, zunehmend flexibilisiert wird. Denn Vernunft entfaltet sich inmitten von pluralen Formen und will im Sinne einer sich offerierenden neuen „Vernünftigkeit" neue Gestaltungsmodi unter Beteiligung aller Mitarbeiter finden, um dadurch Frauen in Führungspositionen zu fördern.

Innerhalb dieses Diskurses soll die Wahrnehmung des Themas auf wirtschafts- und gesellschaftspolitischer Ebene verdichtet werden, indem es gilt, Frauen wie Männer bereits in der Gegenwart für die Gestaltung von Zukunft zu sensibilisieren. Die Multioptionsgesellschaft entfaltet dort ihre gesamten (Wissens-) Potentiale, wo der gesellschaftspolitische Strukturwandel von einem kreativen und produktiven Umgang mit Informationen und Informationstechnik begleitet wird und wo er Einfluss auf plurale Formen von Tätigkeiten sowohl im Erwerbsleben als auch im sozialen und privaten Leben in der Weise nimmt, dass bei allen Tätigkeiten ein Aushandlungsprozess der Betroffenen und der Mitwirkenden unter Berücksichtigung ihrer Interessen sichtbar gemacht und damit zum Gegenstand für Organisationsprozesse wird.

\section{$\mathrm{Zu}$ den einzelnen Kapiteln des Bandes}

Der Band gliedert sich in vier Kapitel, um diese Diskussion innerhalb verschiedener Kontexte jeweils zu vertiefen und teilweise auszuloten.

In Teil I des Bandes konzentrieren sich die Beiträge auf die als wichtig erachtete Diskussion zu Diversity, die aber keine geschlossene Diskussion wiedergibt, sondern innerhalb von gesellschafts- und wirtschaftspolitischen Perspektiven die Vielfalt der Annäherungen an das Thema Frauen und Männer in Führungspositionen aufleuchten lässt, um einen profunden Blick auf das Thema zu gewinnen, bzw. sich mit den Themen in der Vielfalt vertraut machen zu können. Dazu gehören die Beiträge von J. Limbach, S. Peters, N. Bensel, G. Höhler, J. v. Friesen/M. Rühl und B. Schaeffer-Hegel.

Die Teile II und III des Bandes greifen den Untertitel des Bandes auf. In Teil II werden Diversity-Ansätze und Managing-Diversity-Konzepte in ihrem jeweiligen strategischen Diskurs auf der Ebene der Erarbeitung von Konzepten und Strategien vorgestellt, die den 
Problemkreis auf theoretischer Ebene verdeutlichen und für eine entsprechende Wahrnehmung dieser Themen auf gesellschafts- und wirtschaftspolitischer Ebene hinwirken können. Sie zeigen die vielfältigen wechselseitigen Verflechtungen und Kopplungen unterschiedlicher Bereiche. Die Beiträge dazu sind von G. Krell, P. Sepehri/D. Wagner, M. Rühl, N. Gundlach/P. Koseck, K. Heuer und E. Ferrari/F. Rothgängel/E. Sonuç.

In Teil III stehen konkrete best-practice-Beispiele im Vordergrund, in denen vielfältige Konzeptionen für und Erfahrungen von Organisationen diskutiert werden. Sie stehen für eine Vielfalt der Betrachtungen und Schwerpunktsetzungen, die das gesellschaftspolitische Engagement von Organisationen zum Ausdruck bringen, das in den vergangenen Jahren über Netzwerke Anerkennung und Akzeptanz gefunden hat. Insofern wird auf der best-practice-Ebene die Netzwerkstruktur ein wichtiger Parameter. Nicht selten wird behauptet, dass Frauen in Führungspositionen nicht repräsentativ anzutreffen sind, weil sie keinen Zugang zu entscheidenden Netzwerken haben oder sich in Netzwerken von Frauenorganisationen in der Peripherie der jeweiligen Machtzentren aufhalten. Netzwerke sind soziales und immaterielles Kapital und gehören damit zu den Humanressourcen. In diesem Kapitel wird diese Thematik aus unterschiedlichen Perspektiven ausgeleuchtet. Die Beiträge in diesem Teil sind von G. Kuppe/K. Körner, D. Jansen/H. Lukoschat, U. Raue, S. Schönfeld/N. Tschirner und N. Haasen.

Der Teil IV des Bandes widmet sich der empirischen Perspektive von Erfahrungen mit Diversity aus jeweils unterschiedlichen Betrachtungskontexten. Es geht um Einschätzungen und Reflexionen zum Thema, d.h. es geht um Fragen, die die Unterrepräsentanz von Frauen in Führungspositionen als gesellschaftspolitisches Phänomen aus jeweils unterschiedlichen Perspektiven analysieren und Antworten auf diese Fragen suchen. Die einzelnen Beiträge stammen von $\mathrm{M}$. Osterloh/S. Littmann-Wernli, M. E. Domsch/ A. Ladwig, N. Ott, R. Liebold, B. Stieler-Lorenz, B. Seewald, S. Pöhlmann.

Etlichen Personen sind wir zu besonderem Dank für die Unterstützung des vorliegenden Bandes verpflichtet; dieser Dank gilt Sandra Wahlstab, Sandra Dengler, Anja Poppeck, Ulrike Frosch, Katja Althaus und Jana Hofmann an der Otto-von-Guericke-Universität Magdeburg und Katharina Heuer, Katharina Oppolzer und Cornelia Marx von der DaimlerChrysler Services AG in Berlin. 


\section{Inhaltsverzeichnis}

Sibylle Peters / Norbert Bensel

Editorial. 5

\section{Teil I Diversity: Gesellschafts- und wirtschaftspolitische Perspektiven}

Jutta Limbach

Geschlechtergerechtigkeit im 21. Jahrhundert 15

Sibylle Peters

Frauen in Führungspositionen: Der Diskurs über Geschlechterdifferenz

und -arrangements

Norbert Bensel

Auf dem Weg in die Dienstleistungsgesellschaft: Neue Chancen

für Frauen und Männer in der Arbeitswelt

Gertrud Höhler

Geschlechterarrangements im Umbruch: Neue Bündnisse unter Wölfin und Wolf

Juliane v. Friesen / Monika Rühl

Chancengleichheit im Berufsleben: Brauchen wir auch in der

Privatwirtschaft den Gesetzgeber?

Barbara Schaeffer-Hegel

Frauen und Macht heute - Gerechtigkeit für die Generation von morgen

\section{Teil II Diversity-Ansätze und Managing-Diversity- Konzepte im strategischen Diskurs}

Gertraude Krell

Diversity Management: Optionen für (mehr) Frauen in Führungspositionen? .... 105 
Paivand Sepehri / Dieter Wagner

Diversity und Managing Diversity: Verständnisfragen, Zusammenhänge

und theoretische Erkenntnisse.

\section{Monika Rühl}

Diversity in Deutschland in einem globalisierten Unternehmen:

Neuausrichtung des Personalmanagements am Beispiel der Lufthansa

Nicole Gundlach / Pamela Koseck

Diversity Is Much More Than A Workforce Issue

Katharina Heuer

„Managing Diversity“ in einem globalen Unternehmen:

Best-practice-Beispiele bei DaimlerChrysler Services

Elisabeth Ferrari / Friedlinde Rothgängel / Ebrû Sonuç

Das Hütchenspiel

\section{Teil III Best Practice: Diversity-Konzepte für und Erfahrungen von Organisationen im Diskurs}

Gerlinde Kuppe / Kristin Körner

Gender Mainstreaming: Ein Beitrag zum Change Management in Politik und Verwaltung.

Dorothea Jansen / Helga Lukoschat

Netzwerke und Empowerment: Die Europäische Akademie für Frauen in Politik und Wirtschaft Berlin

Ursula Raue

Der Deutsche Juristinnenbund: Ein Frauen-Netzwerk

Simone Schönfeld / Nadja Tschirner

Mentoring-Programme für Frauen - Ein Anstoß zum Aufstieg.

Nele Haasen

Mentoring für Frauen - Faktoren für die erfolgreiche Umsetzung 


\section{Teil IV Reflexionen: Erfahrungen mit Diversity aus unterschiedlichen Perspektiven}

Margit Osterloh / Sabina Littmann-Wernli

Die ,gläserne Decke“ - Realität und Widersprüche 259

Michel E. Domsch / Ariane Ladwig

Doppelkarrierepaare und neue Karrierekonzepte: Eine theoretische und empirische Ausschnittsuntersuchung

Notburga Ott

The Economics of Gender: Gedanken zu Work-Life-Balance

Renate Liebold

Die Vereinbarkeit von Beruf und Familie aus männlicher Sicht:

Ein Fallbeispiel

Brigitte Stieler-Lorenz

Management von leanen und digital vernetzten Unternehmen:

Change Management zwischen Chancen, Fallen, Perspektiven

Beate Seewald

Die Entwicklung einer medizinischen Rehabilitationsklinik:

Skizze einer Change-Managerin

Simone Pöhlmann

Zwischen Konflikt und Konsens: Streiten lernen für innovative

Management-Strategien 